\title{
Research on Clinical Effect Observation of Thoracoscopy in the Treatment of Early Non - Small Cell Lung Cancer
}

\author{
Jie $\mathrm{Ma}^{1, \mathrm{a}}$ \\ ${ }^{1}$ Nanning Fourth People's Hospital, Nanning, Guangxi, China, 530021 \\ aemail,
}

Keywords: Early Non-Small Cell Lung Cancer; Thoracoscopy; Thoracotomy; Treatment Effect

\begin{abstract}
This paper analysis the patients with early non - small cell lung cancer treated by thoracoscopy. Methods: 96 patients with non-small cell lung cancer treated in our hospital from 2004 to 2016 were divided into control group and study group according to the random assignment method. The thoracic surgery was performed in the control group and the thoracic surgery was performed in the control group. The therapeutic effect of the two groups was compared. Results: Compared with the two groups, there was no significant difference in the number of lymph node dissection between the two groups ( $\mathrm{P}>0.05$ ). There was no significant difference between the two groups in the amount of bleeding, drainage time and postoperative pain time is better than thoracotomy surgery group, the two groups were different $\mathrm{P}<0.05$ and it has statistically significant. Conclusion: Thoracoscopic surgery for early non-small cell lung cancer (NSCLC) is effective for lymph node dissection, and it has the characteristics of less trauma, less complication, less hospital stay and less healing time. It is worthy of clinical application.
\end{abstract}

\section{Introduction}

Lung cancer is one of the fastest growing morbidity and mortality, one of the greatest malignant tumors of human health and life. The incidence and mortality of lung cancer in China are among the highest in all malignancies. The incidence of non-small cell lung cancer in various pathologic types of lung cancer is more than $80 \%$. [1] Compared with small cell carcinoma, the growth of cancer cells is slower and the diffusion is relatively later, and about $75 \%$ of patients Has been in the late, 5-year survival rate is very low For early non-small cell lung cancer lymph node dissection, to a certain extent, reduce the rate of metastasis [2]. With the rapid application of thoracoscopy in clinical practice in recent years, in the treatment of lung cancer also achieved good therapeutic effect, this paper for patients with non-small cell lung cancer in clinical use of different treatment methods to study the different surgical programs for Patient treatment and intraoperative and postoperative response, the specific summary is as follows.

\section{Materials and Methods}

Clinical Data. In this study, 96 patients with non-small cell lung cancer (NSCLC) underwent surgical treatment from 2004 to 2016 were enrolled in this study. All patients were diagnosed as non-small cell lung cancer after admission. 96 patients were divided into control group and study group according to the random assignment method. There were 32 cases of male and female 16 cases, age $(49 \sim 68)$ years, mean age $(46.5 \pm 26.9)$ Year, of which TNM staging 42 cases I , 6 cases II. Pathological types of lung cancer in 12 cases of squamous cell carcinoma, squamous cell carcinoma in 16 cases, 20 cases of adenocarcinoma Lesion location, left upper lobe and left lower lobe of the 9 cases and 11 cases, 19 cases of right upper lobe, right lower lobe 11 cases. In the study group, 29 males and 19 females had age (53-71 years) and mean age (47.2 \pm 27.1 ) years, among which 43 patients had TNM stage I and 5 cases. Lung cancer type adenosquatous carcinoma in 10 cases, squamous cell carcinoma in 15 cases, 23 cases of adenocarcinoma Lesion location, left upper lobe and left lower lobe of the 6 cases and 8 cases, 20 cases of right upper lung, right lower lobe in 14 cases. There was no significant difference in age, sex, TNM staging and location and type 
of lung cancer between the two groups, $\mathrm{P}>0.05$ was not statistically significant and comparable.

Treatment. Two groups of patients were treated with different surgical methods, in which the control group using thoracotomy, the study group for thoracoscopic surgery.

The control group of patients with thoracotomy, the patient first patients needs to use supine position, double-lumen endotracheal intubation and systemic intravenous anesthesia, after the lateral incision, cut the hilar mediastinal pleura to the ipsilateral hilar direction of anatomy, free venous veins, if bronchial lymphadenopathy, suspected to have metastases, this time should be cut off the veins. At the top of the pleura, cut the pleura, the subclavian artery and the head arm artery revealed, to confirm the ipsilateral recurrent laryngeal nerve, down the free, the head arm arterial stem, superior vena cava, tracheal fascia revealed, diaphragmatic adipose tissue into the superior vena cava. At the same time according to this order in turn mediastinal lymph nodes, tracheal lymph nodes, tracheal lymph nodes, tracheal bronchial lymph nodes, tracheal lymph nodes were cleaned. In addition to the bulge of the lymph nodes and the ipsilateral bronchial lymph node dissection, you need to pull the lungs in front of the exposed at the same time to protect the vagus nerve, cut off the vagus nerve plexus, showing the removal of lymph nodes after pericardium. Finally, from the lowest point of the lung ligament dissection of the lung ligament lymph nodes, continue to free up, clean esophageal lymph nodes.

The patients underwent thoracoscopic mediastinal lymphadenectomy. First patients take lateral position, for double-lumen endotracheal intubation and systemic anesthesia. And then thoracoscopic resection of lung cancer, usually in the 6th or 7th intercostal axillary line, the thoracoscopic camera system into the casing, and then the first 7 or 8 intercostal axillary line for an operation hole. A 5 to $7 \mathrm{~cm}$ incision was made along the fourth intercostal space for placing the stump closure and removing the specimen. Thoracoscopic instruments are used for thoracic operation, in accordance with the traditional way of branching free pulmonary blood vessels, bronchus. Cut the stapler to nail the pulmonary vessels, the bronchus. The hilar and mediastinal lymph nodes are cleaned according to conventional methods. If the patient's lesion is in the middle or upper right, at least two lymph nodes need to be cleaned at the same time.

Indicators of Observation. The operation time, intraoperative blood loss, retention time of drainage tube, postoperative analgesia time and postoperative hospital stay were observed.

Statistical Analysis. In this study, we used software SPSS20.0 for data processing. All data were tested with $\mathrm{t}$, and the data were counted as $\mathrm{x} 2$. The data were expressed as\% .p <0.05 was statistically significant.

\section{Results}

Two groups of patients were successful operation and patients with higher survival after surgery. The operative time and the number of lymph nodes were compared between the two groups. There was no significant difference between the two groups in the operation time $(\mathrm{P}>0.05)$. $(265 \pm 33) \mathrm{ml}$, the amount of bleeding in the study group (160.6 \pm 11.1$)$ compared with the two groups, the study group was less than the control group, the difference was $\mathrm{P}<0.05$ was statistically significant. Drainage time, postoperative pain time, hospitalization time comparison, significant thoracoscopic surgery group shorter than thoracotomy group, the difference was $\mathrm{P}<0.05$ was statistically significant. (See Table 1) 
Table I:

\begin{tabular}{lllllll}
\hline Group & $\begin{array}{l}\text { Operation } \\
\text { Time (min) }\end{array}$ & $\begin{array}{l}\text { Intraoperative } \\
\text { blood loss (ml) }\end{array}$ & $\begin{array}{l}\text { Number of } \\
\text { lymph } \\
\text { node } \\
\text { dissection }\end{array}$ & $\begin{array}{l}\text { Drainage } \\
\text { tube } \\
\text { retention } \\
\text { time (d) }\end{array}$ & $\begin{array}{l}\text { Postoperativ } \\
\text { e analgesia } \\
\text { time (d) }\end{array}$ & $\begin{array}{l}\text { Hospitalization } \\
\text { time (d) }\end{array}$ \\
\hline $\begin{array}{l}\text { Control } \\
\text { Group }\end{array}$ & $108 \pm 32$ & $265 \pm 33$ & $14.1 \pm 2.4$ & $4.7 \pm 3.3$ & $4.1 \pm 3.3$ & $16.5 \pm 5.7$ \\
\hline $\begin{array}{l}\text { Research } \\
\text { Group }\end{array}$ & $104 \pm 34$ & $160 \pm 11.1$ & $13.3 \pm 3.1$ & $2.3 \pm 1.6$ & $1.7 \pm 2.5$ & $7.8 \pm 3.2$ \\
\hline
\end{tabular}

\section{Discussion}

Surgery is the core of early non-small cell lung cancer treatment, according to research reports for such patients with surgery-based program survival rate is high, and chemotherapy alone or radiotherapy in patients with low survival rate.

Although the traditional thoracotomy surgery can meet the needs of the surgical field of vision, but from the posterior lateral incision of the chest, the incision is large, and in the incision need to cut off a large number of large muscles, bleeding volume, thoracotomy and postoperative clearance Chest is more complex, longer operation time, patients prone to "frozen shoulder" and the upper limb on the difficult situation, recovery time is longer, affecting the normal life of patients work. With the development of thoracic surgery, people become more and more comprehensive understanding of minimally invasive surgery, minimally invasive surgery can reduce the trauma, less intraoperative blood loss, compared to traditional surgery for other patients with less organ damage, postoperative patient body Fast recovery, so thoracoscopic surgery as early non-small cell lung cancer treatment of choice. Although there are so many advantages of thoracoscopic surgery, but still exist in the learning curve is longer, higher requirements of equipment and equipment, some of the more complex operation is difficult to complete in the mirror.

Through this study can be summed up, thoracoscopic surgery and traditional thoracotomy for early non-small cell lung cancer, the two groups of patients with surgical time, the number of lymph node dissection and so no significant difference, but intraoperative blood loss, postoperative analgesia time and Hospitalization time and other comparison, thoracoscopic surgery was significantly better than thoracotomy surgery group, the two groups were different $\mathrm{P}<0.05$ was statistically significant. In summary, the treatment of early non-small cell lung cancer patients with thoracoscopic surgery, the effect is not inferior to thoracotomy, postoperative rehabilitation is fast, is conducive to improving the quality of life of patients, worthy of clinical application.

\section{References}

[1] Jiang Wei, Xi Junjie, Wang Hao, Ge Di, Tan Lijie, Fan Hong, Xu Songtao, Xu Zhenglang, Wang Qun. The efficacy of total thoracoscopic lobectomy in the treatment of early non-small cell lung cancer[J]. Journal of Cardiovascular Surgery, 2012, 11(2): 120-124.

[2] Tan Qunyou, Deng Bo, Kang Puming, Zhou Jinghai, He Yong, Wang Ruwen, Jiang Yaoguang. 276 cases of single-hole full-thoracoscopic lobectomy for early non-small cell lung cancer in the perioperative period[J] .Journal of the Third Military Medical University Journal, 2012, 21(5): 2181-2183.

[3] Liu Zongliang. Thoracoscopy and thoracotomy surgery for early non-small cell lung cancer clinical efficacy observation[J]. Contemporary Medicine, 2010, 26(5): 107-108.

[4] BuikhuisenWienekeA, ScharpfeneckerMarion, GriffioenArjanW, Korse Catharina M, vanTinterenHarm, Baas Paul. A Randomized Phase II Study Adding Axitinib to Pemetrexed-Cisplatin in Patients with Malignant Pleural Mesothelioma: A Single-Center Trial 
Combining Clinical and Translational Outcomes [J]. Journal of thoracic oncology: official publication of the International Association for the Study of Lung Cancer, 2016, 115(1):45-48.

[5] Gao Ke, Liu Zaoyang, Wu Jian, Li Hailin, Xiao Pang, Wang Xiaohui, Chen Jian, Wang Tao. Clinical effect of one-way thoracoscopic lobectomy for early non-small cell lung cancer[J]. Journal of Vascular Surgery, 2011, 7(3): 231-235. 